\title{
A Prospective Study of the Prevalence of the Polycystic Ovary Syndrome in Unselected Caucasian Women from Spain*
}

\author{
MIRYAM ASUNCIÓN, ROSA M. CALVO, JOSÉ L. SAN MILLÁN, JOSÉ SANCHO, \\ SERGIO AVILA, AND HÉCTOR F. ESCOBAR-MORREALE \\ Departments of Endocrinology (M.A., R.M.C., J.S., H.F.E.-M.), Molecular Genetics (J.L.S.M.), and \\ Biochemistry (S.A.), Hospital Ramón y Cajal, Madrid 28034, Spain
}

\begin{abstract}
We prospectively estimated the prevalence of the polycystic ovary syndrome (PCOS), as defined by the NIH/NICHHD 1990 endocrine criteria, in a population of 154 Caucasian women of reproductive age reporting spontaneously for blood donation. Anthropometric data; the presence of hirsutism, acne, and androgenic alopecia; and the menstrual history were recorded by a single investigator. In 145 women, blood samples were also obtained for measurement of serum androgen levels. PCOS was defined by the presence of 1) oligomenorrhea, 2) clinical and/or biochemical hyperandrogenism, and 3) exclusion of hyperprolactinemia, thyroid disorders, and nonclassic 21-hydroxylase deficiency. Hirsutism was defined by a modified FerrimanGallwey score of 8 or more, acne was considered as a sign of hyperan-
\end{abstract}

drogenism when persistent after the second decade of life, and hyperandrogenemia was defined by an increase in circulating testosterone or dehydroepiandrosterone sulfate or an increase in the free androgen index above the 95th percentile of the control values derived from the nonhirsute, nonacneic women having regular menses who were not receiving hormonal therapy. PCOS was present in $10(6.5 \%)$, hirsutism was present in $11(7.1 \%)$, and acne was present in 19 $(12.3 \%)$ of the 154 women. Our results demonstrate a $6.5 \%$ prevalence of PCOS, as defined, in a minimally biased population of Caucasian women from Spain. The polycystic ovary syndrome, hirsutism, and acne are common endocrine disorders in women. (J Clin Endocrinol Metab 85: 2434-2438, 2000)
$\mathrm{T}$ HE POLYCYSTIC ovary syndrome (PCOS) appears to be one of the most common endocrine disorders of women (1). Moreover, PCOS is associated with significant reproductive, endocrine, metabolic, and cardiovascular, morbidity (2). However, the data regarding the prevalence of this disorder are surprisingly scarce and are biased by the different criteria used to define the disease. When detected by sonography, polycystic ovaries are present in $14-23 \%$ of unselected women (3-6). However, a significant proportion of these patients are asymptomatic (7), whereas not all patients with hyperandrogenic anovulation have polycystic ovaries on ultrasound examination (6-9), suggesting that ultrasound is a nonspecific marker for the diagnosis of PCOS.

At present, the diagnosis of PCOS is usually based on the criteria derived from the $1990 \mathrm{NIH} / \mathrm{NICHHD}$ conference (10): 1) menstrual dysfunction, 2) clinical hyperandrogenism (hirsutism, acne, androgenic alopecia) and/or hyperandrogenemia, and 3) exclusion of other related disorders, such as hyperprolactinemia, nonclassic adrenal hyperplasia, or thyroid disease. Using these criteria, Knochenhauer et al. (11) reported a $4.0 \%$ prevalence of PCOS in the southeastern U.S., namely, Birmingham, AL. This carefully designed study in-

Received September 8, 1999. Revision received January 13, 2000. Rerevision received March 16, 2000. Accepted March 19, 2000.

Address all correspondence and requests for reprints to: Héctor F. Escobar-Morreale, M.D., Ph.D., Department of Endocrinology, Hospital Ramón y Cajal, Carretera de Colmenar Km. 9,100, 28034 Madrid, Spain.

* This work was supported in part by a grant (Proyecto 08.6/0022/ 1998) from the Consejería de Investigación y Cultura, Comunidad de Madrid, Spain, and grants (FIS 00/0414 and FIS 98/3044) from the Fondo de Investigación Sanitaria, Ministerio de Sanidad y Consumo, Spain. volved both White and Black women and avoided any significant bias in the selection of the participants. Further, a similar $6.8 \%$ prevalence of PCOS has been recently reported in the Greek island of Lesbos (12).

In the present study we estimated the prevalence of PCOS and hirsutism in 154 consecutive Caucasian women from Madrid, Spain, using a design similar to that used by Knochenhauer et al. (11). To avoid any selection of the population, all of the women studied here were consecutive blood donors reporting spontaneously to our hospital for that reason.

\section{Subjects and protocol}

\section{Subjects and Methods}

The study population consisted in Caucasian female blood donors reporting to the Department of Hematology of the Hospital Ramón y Cajal from May to June 1999. The presence of hirsutism was scored in every woman by a single investigator, using a modification of the Ferriman-Gallwey method (13), quantitating the presence of terminal hairs over nine body areas (i.e. upper lip, chin, chest, upper and lower abdomen, upper and lower back, upper arms, and thighs). The presence or absence of acne and androgenic alopecia was recorded, and weight, height, and waist and hip circumferences were measured.

A history form was completed, including menstrual dating and irregularity, hirsutism and acne, reproductive history, gynecological history, use of medication including oral contraceptive pills, and family history of male pattern premature baldness, carbohydrate intolerance, and arterial hypertension. In women who were receiving hormonal therapy, their menstrual history before treatment and the reason for treatment were recorded. Women with physiological menopausepresent or past symptoms of hypoestrogenism such as hot flushes and vaginal dryness, emotional lability, and menstrual abnormalities in a woman older than $45 \mathrm{yr}(14)$ - were excluded from the study. None of the subjects was younger than $18 \mathrm{yr}$ old, which is the minimum legal age 
for blood donation in Spain. In those women who were found to be suitable blood donors, serum samples were obtained, and were stored at $-20 \mathrm{C}$ until assayed. When women were finally not accepted as blood donors because of present anemia or previous history infectious hepatitis, no serum samples were obtained.

The study was approved by the institutional review board of the Hospital Ramón y Cajal, and written informed consent was obtained from all the subjects.

\section{Assays}

Serum samples were analyzed for total testosterone $(\mathrm{T})$, sex hormonebinding globulin (SHBG), dehydroepiandrosterone sulfate (DHEAS), progesterone $\left(\mathrm{P}_{4}\right), \mathrm{LH}, \mathrm{FSH}$, and estradiol $\left(\mathrm{E}_{2}\right)$. In women who matched the criteria for PCOS, PRL, TSH, and 17-hydroxyprogesterone (17-OHP) were also measured to exclude hyperprolactinemia, hypothyroidism, and nonclassic 21-hydroxylase deficiency, respectively.

Total T, SHBG, DHEAS, $\mathrm{P}_{4}$, LH, FSH, $\mathrm{E}_{2}$, PRL, and TSH were measured, each hormone in a single assay, using an automated immunochemiluminescence method (Immulite, Diagnostic Products, Los Angeles, CA). 17-OHP was measured by direct RIA using a commercially available kit (ImmuChem, ICN Biomedicals, Inc., Costa Mesa, CA) in a single assay. The mean intraassay coefficients of variation were as follows: $7.4 \%$ for $\mathrm{T}, 6.5 \%$ for SHBG, $6.9 \%$ for DHEAS, $8.2 \%$ for $\mathrm{P}_{4}, 5.7 \%$ for $\mathrm{LH}, 6.4 \%$ for $\mathrm{FSH}, 9.3 \%$ for $\mathrm{E}_{2}, 6.2 \%$ for PRL, $7.1 \%$ for TSH, and $9.5 \%$ for 17-OHP. The free androgen index (FAI) was calculated using the formula [T $(\mathrm{nmol} / \mathrm{L}) \times 100] /$ SHBG $(\mathrm{nmol} / \mathrm{L})]$.

\section{Criteria for the definition of PCOS}

As stated above, PCOS was defined by the presence of 1) menstrual dysfunction, 2) clinical hyperandrogenism and/or hyperandrogenemia, and 3) exclusion of other disorders (10). Menstrual dysfunction was considered when the women had oligomenorrhea, defined by more than six cycles with a length of more than 35 days, and/or when the patient had not had any menstrual bleeding for 3 consecutive months during the last year. Clinical hyperandrogenism was defined by the presence of hirsutism, represented by a hirsutism score of 8 or more, persistence of acne during the third decade of life or later, or the presence of androgenic alopecia. No attempts were made to grade the severity of acne or alopecia. Hyperandrogenemia was defined by a circulating $\mathrm{T}$ and DHEAS levels and / or FAI higher than the 95th percentile for the women studied here who had no clinical evidence of hyperandrogenism or menstrual disturbances and who were not taking hormonal medication nor had had previous oophorectomy or hysterectomy. Hyperprolactinemia, thyroid dysfunction, and nonclassic 21-hydroxylase deficiency were excluded in all of the women who achieved the other criteria for the diagnosis of PCOS.

\section{Statistical analysis}

The results are expressed as the mean \pm SD in the text and tables. One-way ANOVA followed by the least significant difference test were used to compare the means of the different groups of women. A $\chi^{2}$ test was used for discontinuous variables. $P<0.05$ was considered significant. A statistical software package was used to perform the analyses (SPSS for the Macintosh, version 6.1.1, SPSS, Inc., Chicago, IL).

\section{Results}

One hundred and fifty-four women agreed to participate in the study. Clinical variables were evaluated in all of them, including hirsutism scores and menstrual history, and in 145 women serum samples were also obtained. Mean age, body mass index , and waist to hip ratios were $33.1 \pm 9.1 \mathrm{yr}, 23.8 \pm$ $3.2 \mathrm{~kg} / \mathrm{m}^{2}$, and $0.79 \pm 0.06$, respectively. Eight women $(5.2 \%)$ showed a hirsutism score of 8 or more, and 3 other subjects, with hirsutism scores of 2,3, and 5, were receiving hormonal treatment for hirsutism. Therefore, 11 of the 154 women $(7.1 \%)$ may be considered to have hirsutism. None of the other subjects had a history of treated hirsutism. Persistence of acne after the second decade of life was found in 19 women $(12.3 \%)$. None of the women had androgenic alopecia.

Thirty-one women were taking oral contraceptive pills, including those 3 patients being treated for hirsutism and another 3 subjects with a hirsutism score of 8 or more who were taking the pill solely as contraceptive. Seventy-nine women did not have hirsutism, acne, or menstrual disturbances; were not receiving hormonal therapy, and had no history of previous hysterectomy or oophorectomy. These women composed the normal population from which the upper control 95th percentile values for androgens were calculated: T, $2.5 \mathrm{nmol} / \mathrm{L}$; FAI, 3.9; and DHEAS, 11.9 $\mu \mathrm{mol} / \mathrm{L}$.

Of the whole population studied, 30 women (19.5\%) had a history consistent with oligomenorrhea, and 13 fulfilled the 2 first criteria (oligomenorrhea and evidence of hyperandrogenism) for the diagnosis of PCOS. However, hyperprolactinemia, defined by serum PRL more than $24 \mu \mathrm{g} / \mathrm{L}$, was present in 3 of these women (serum PRL levels were 26.8, 50.8 , and $80.4 \mu \mathrm{g} / \mathrm{L}$, respectively), and therefore, the diagnosis of PCOS was sustained in only 10 of the 154 subjects studied $(6.5 \%)$. In 9 of the 10 patients diagnosed with PCOS, serum samples were available. None of these women had 17-OHP levels above $6.0 \mathrm{nmol} / \mathrm{L}$, which is the cut-off level suggested for maximum sensitivity in the detection of nonclassic adrenal hyperplasia using 17-OHP measurements obtained from nonearly morning samples (15). Further, none of these patients had abnormal serum TSH levels, ruling out thyroid dysfunction.

The clinical characteristics of the 10 patients who met all of the criteria for the diagnosis of PCOS are described in Table 1. The diagnosis of PCOS was based on the presence of oligomenorrhea and hirsutism in 4 of the 10 patients. Hyperandrogenemia was present in 1 of them, who also presented acne; another patient was taking oral contraceptive pills, and her normal androgen levels might represent the effect of such treatment on ovarian function. In another patient, serum androgen levels were not measured. The diagnosis of PCOS was solely sustained on the basis of oligomenorrhea and hyperandrogenemia in 4 patients ( 1 of these women had acne, but was 18 yr old) and of oligomenorrhea, acne, and hyperandrogenemia in 2 patients.

In addition to 7 of the women diagnosed with PCOS and the women with hyperprolactinemia, hyperandrogenemia was present in another 19 women who did not have oligomenorrhea. Thirteen of them had no clinical manifestations of hyperandrogenism, but 1 of these women was taking oral contraceptive medication. Five had acne (1 was taking a contraceptive pill), and 1 had hirsutism.

As stated above, of the 11 patients considered to have hirsutism, 4 were diagnosed as having PCOS, 2 subjects had oligomenorrhea and hyperprolactinemia, and 5 had hirsutism with regular menstrual cycles. Of the latter, hyperandrogenemia was present in only 1 woman, but another 3 were taking oral contraceptive pills, which may have lowered their androgen concentrations.

We then compared the clinical data and serum androgen concentrations of the women diagnosed with PCOS with those of 1) the women with hyperandrogenemia and regular menstrual cycles, 2) the women who presented with idio- 
TABLE 1. Characteristics of women diagnosed with PCOS

\begin{tabular}{|c|c|c|c|c|c|c|c|c|c|c|}
\hline $\begin{array}{c}\text { Donor } \\
\text { no. }\end{array}$ & $\begin{array}{l}\text { Age } \\
\text { (yr) }\end{array}$ & $\begin{array}{c}\text { BMI } \\
\left(\mathrm{kg} / \mathrm{m}^{2}\right)\end{array}$ & $\begin{array}{l}\text { Waist to } \\
\text { hip ratio }\end{array}$ & $\begin{array}{l}\text { Hirsutism } \\
\text { score }\end{array}$ & Acne & Oligomenorrhea & $\mathrm{T}(\mathrm{nmol} / \mathrm{L})$ & FAI & $\begin{array}{l}\text { DHEAS } \\
(\mu \mathrm{mol} / \mathrm{L})\end{array}$ & $\mathrm{OC}$ \\
\hline 15 & 24 & 22.3 & 0.80 & 18 & No & Yes & ND & ND & ND & No \\
\hline 37 & 18 & 23.4 & 0.87 & 1 & Yes & Yes & 1.2 & $5.1^{a}$ & $15.6^{a}$ & No \\
\hline 49 & 22 & 32.9 & 0.82 & 17 & Yes & Yes & $2.9^{a}$ & $17.0^{a}$ & $12.2^{a}$ & No \\
\hline 74 & 30 & 27.5 & 0.88 & 3 & Yes & Yes & 1.3 & $6.7^{a}$ & 8.6 & No \\
\hline 105 & 22 & 25.3 & 0.79 & 6 & No & Yes & 1.1 & $4.5^{a}$ & 5.8 & No \\
\hline 110 & 27 & 24.2 & 0.84 & 21 & No & Yes & $<0.7$ & 2.1 & 6.9 & No \\
\hline 115 & 23 & 19.3 & 0.77 & 9 & No & Yes & $<0.7$ & 0.5 & 6.2 & Yes \\
\hline 116 & 34 & 20.6 & 0.82 & 1 & Yes & Yes & 1.8 & 2.4 & $12.1^{a}$ & No \\
\hline 118 & 20 & 20.9 & 0.69 & 1 & No & Yes & 1.4 & 3.7 & $15.3^{a}$ & No \\
\hline 149 & 27 & 29.1 & 0.73 & 4 & No & Yes & $3.1^{a}$ & $11.0^{a}$ & $16.8^{a}$ & No \\
\hline
\end{tabular}

BMI, Body mass index; T, circulating total testosterone; FAI, free androgen index; DHEAS serum dehydroepiandrosterone sulfate levels; OC, oral contraceptive pills; ND, not determined (blood samples from this woman were not obtained).

${ }^{a}$ Greater than the upper control 95 th percentile value.

pathic hirsutism and/or acne but did not have oligomenorrhea or hyperandrogenemia, and 3) the women without clinical hyperandrogenism who had normal serum androgen levels, representing the nonhyperandrogenic group. This group of nonhyperandrogenic women did not completely match the group of women used to define the normal ranges for serum androgens. The latter was selected on a clinical basis before knowing the serum androgen concentrations, and therefore, some of these women actually had increased levels of the androgens measured. These subjects were included in the group of women with hyperandrogenemia and regular menstrual cycles for this analysis. Conversely, some women with nonhyperandrogenic oligomenorrhea, who could not be used to define the normal ranges for serum androgens, were included in the group of nonhyperandrogenic women. Because of the profound effects of oral contraceptives on serum androgen and SHBG levels, women taking these medications were excluded from these comparisons. Also, the three patients in whom PCOS was excluded because of hyperprolactinemia were not included in this analysis.

Women with PCOS and women with hyperandrogenemia and regular cycles were younger than women with idiopathic hirsutism and/or acne without oligomenorrhea or hyperandrogenemia and nonhyperandrogenic women (Table 2). The higher hirsutism scores were found in women with PCOS, but as a group, women with idiopathic hirsutism and/or acne without oligomenorrhea or hyperandrogenemia had also higher hirsutism scores than nonhyperandrogenic women (Table 2). There were no differences among the groups in body mass index, waist to hip ratios, ages at menarche and pubarche, and number of abortions (Table 2). On the contrary, women with hyperandrogenemia and regular cycles had fewer pregnancies and infants born than the nonhyperandrogenic group or the women with idiopathic hirsutism and/or acne without oligomenorrhea or hyperandrogenemia (Table 2).

Women with hyperandrogenemia and regular cycles had higher $\mathrm{T}$ levels than any of the other groups (Table 2). Serum $\mathrm{T}$ concentrations were also increased in PCOS patients compared to women with idiopathic hirsutism and / or acne without oligomenorrhea or hyperandrogenemia and to nonhyperandrogenic women (Table 2). The FAI and serum DHEAS levels were increased to a similar extent in women with hyperandrogenemia and regular cycles and in PCOS patients compared to patients with women with idiopathic hirsutism and/or acne without oligomenorrhea or hyperandrogenemia and to nonhyperandrogenic women (Table 2). Women with PCOS had decreased SHBG levels compared to women with idiopathic hirsutism and/or acne without oligomenorrhea or hyperandrogenemia and to nonhyperandrogenic women. There were no differences in serum $\mathrm{LH}, \mathrm{FSH}, \mathrm{E}_{2}$, and $\mathrm{P}_{4}$ levels among the groups (Table 2).

Finally, none of the subjects with hyperandrogenic disorders had a history of carbohydrate intolerance or hypertension (their blood pressure measurements were normal at the time of the study), but no further effort was made to diagnose these disorders. Moreover, no significant differences were found among the groups of subjects in the proportion of women with family history of diabetes mellitus, arterial hypertension, or male pattern premature baldness (Table 2).

\section{Discussion}

Although PCOS is believed to be one of the most common endocrine disorders of women $(1,2)$, there are very few data regarding its prevalence in the female population. As stated above, the present endocrine definition of PCOS is based on several criteria, accepted by the NIH/NICHHD-sponsored conference in 1990 (10). To our knowledge, there are only two recent studies estimating the prevalence of PCOS using these criteria. Knochenhauer et al. (11) reported an overall 4.0\% prevalence of PCOS in women from the U.S. (4.7\% in White women and 3.4\% in Black women), and Diamanti-Kandarakis et al. reported a $6.8 \%$ prevalence of PCOS in the Greek island of Lesbos (12).

Using strict NIH/NICHHD criteria, we found a $6.5 \%$ prevalence of PCOS in Caucasian women from Madrid, Spain. However, moderate increases in serum PRL levels are frequently found in women with hyperandrogenism and may also result from the stress of blood sampling and from treatment with oral contraceptives. Without excluding the three patients who were considered as not having PCOS because of mild to moderate hyperprolactinemia, the prevalence of PCOS in our series might have been as high as $8.4 \%$.

Our study share many characteristics with that by Knochenhauer et al. (11), allowing direct comparison between their results and our present findings. Considering 
TABLE 2. Clinical data and serum androgen concentrations of women diagnosed with PCOS as compared to women with hyperandrogenemia and regular menses, women who presented with idiopathic hirsutism and/or acne, but did not have oligomenorrhea or hyperandrogenemia, and nonhyperandrogenic women, excluding women taking oral contraceptives

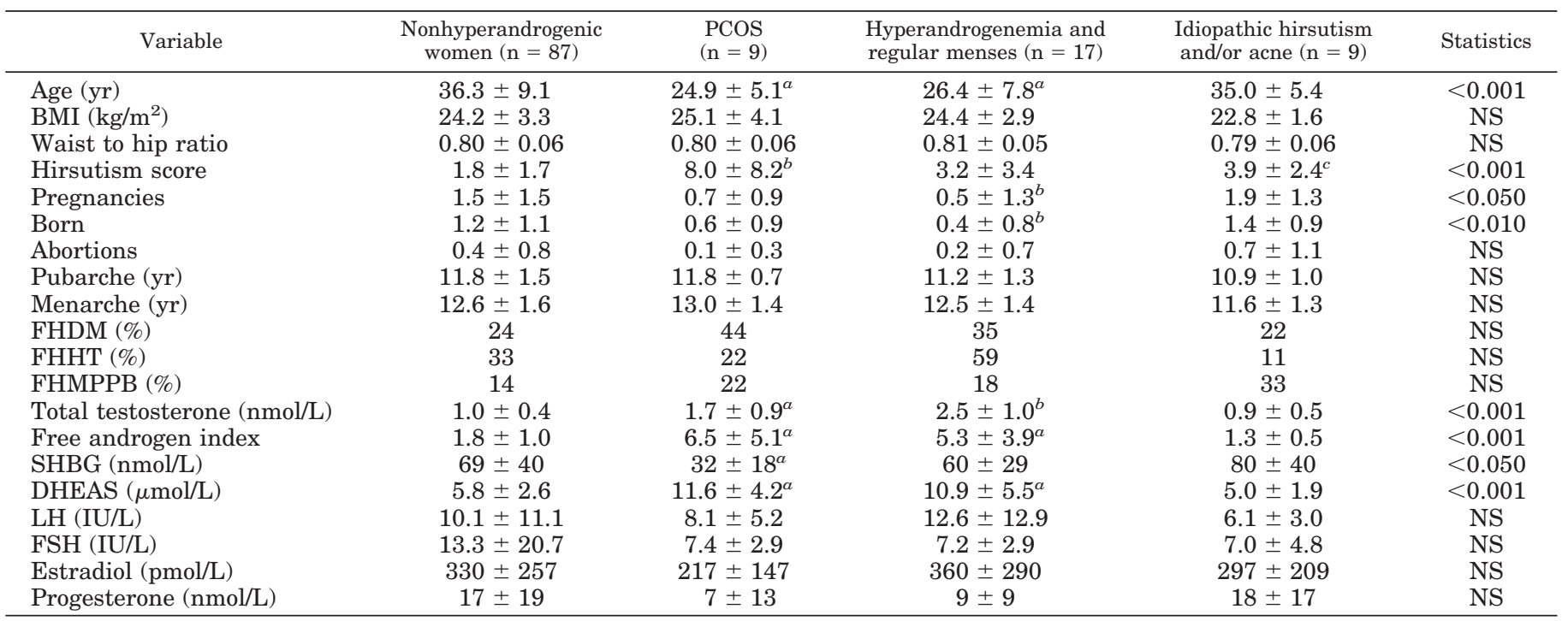

BMI, Body mass index; FHDM, percentage of subjects with family history of diabetes mellitus; FHHT, percentage of subjects with family history of arterial hypertension; FHMPPB, percentage of subjects with family history of male pattern premature baldness; SHBG, circulating sex hormone-binding globulin levels; DHEAS, serum dehydroepiandrosterone sulfate levels. Because of the profound effects of oral contraceptives on serum androgens and SHBG, women taking these medications were excluded for comparisons between groups. Serum samples were obtained from 80 women from the nonhyperandrogenic group, 8 women from the PCOS group, 17 women from the hyperandrogenemia group, and 8 women from the idiopathic hirsutism and/or acne group, who did not have oligomenorrhea or hyperandrogenemia.

${ }^{a}$ Compared to the group of nonhyperandrogenic women and to women with idiopathic hirsutism and/or acne who did not have oligomenorrhea or hyperandrogenemia.

${ }^{b}$ Compared to all the other groups.

${ }^{c}$ Compared to the group of nonhyperandrogenic women.

that all of the women who entered our present study were Caucasian, the size of the sample we studied was similar to the number of White women included in the study from Knochenhauer et al. (11) and was adequate to estimate the prevalence of PCOS as previously discussed (11). Moreover, both studies were designed to minimize bias of the sample population studied.

We prospectively included consecutive female blood donors attending our hospital during a limited period of time. We believe that blood donors are an especially adequate sample of the population for several reasons: 1) they were usually healthy people, not seeking medical care for any reason at the time of the study; 2) as in Spain blood donation is not rewarded in any way, there is no bias derived from the socio-economic background; 3) blood donors have mixed ethnic origins, representing the population where they live; 4) there is no preselection of blood donors, as they come to the hospital without scheduling; and 5) it is easy to obtain blood samples from them. Finally, as in the study from Knochenhauer et al. (11), we defined the normative data for serum androgens from the group of nonhirsute, nonacneic, eumenorrheic women of the study population who were not taking hormonal preparations. On the contrary, as occurred to Knochenhauer et al. (11) in their study, we were not able to control precisely for the time of the day of serum sampling or for the day of the menstrual cycle. However, as elegantly discussed by Knochenhauer et al. (11), none of these factors has been demonstrated to have a significant impact on the clinical value of circulating androgens.
Madrid is representative of the population of Spain, as its present population was conformed during the last $50 \mathrm{yr}$ by immigrants from all areas of the country. All of the women studied here were Caucasian, although it should be noted that the population from Spain has important Jewish and Arabic influences dating back to the Middle Ages, as occurs with most Mediterranean countries.

Minor differences in the design of the study may explain the slightly higher prevalence of PCOS found in Spain $(6.5 \%)$ compared to White women from Alabama (4.7\%). We defined clinical hyperandrogenism by the presence of hirsutism and/or acne, whereas Knochenhauer et al. (11) only considered hirsutism for the diagnosis of PCOS. Although in none of our PCOS patients did the diagnosis rely solely on acne and oligomenorrhea, in the study by Knochenhauer et al. (11), the inclusion of acne as a sign of hyperandrogenism would have increased the prevalence of PCOS in White women to $5.4 \%$. Further, we used less stringent criteria for the definition of oligomenorrhea, which might be responsible for the $1 \%$ increase in the prevalence of PCOS in Spain compared to Alabama.

However, we found higher prevalences of hirsutism, as defined by a hirsutism score of 8 or more, and acne in Spanish women $(7.1 \%$ and $12.3 \%$, respectively) compared to White women from Alabama (2.8\% and 5.2\%, respectively) (11), suggesting that hyperandrogenic disorders might be more prevalent in Spain. As reviewed by Knochenhauer et al. (11), older studies have reported excess "hairiness" in $5-15 \%$ of consecutive Caucasian women (16-20), and Ferriman and 
Gallwey (21) found that only $5 \%$ of the general population had a hirsutism score of 6 or greater in the original study. The prevalence of hirsutism in our series does not appear to be exaggerated with respect to these previous studies.

As stated above, Diamanti-Kandarakis et al. (12) recently found a $6.8 \%$ prevalence of PCOS in the Greek island of Lesbos. However, this study, which in addition to evaluating the prevalence of PCOS was designed to evaluate hormonal and metabolic abnormalities and family history of metabolic disorders in these patients, presented significant methodological differences compared to our present study and that by Knochenhauer et al. (11). First, instead of studying unselected women, the sample of the female population of Lesbos was recruited after a large scale informational campaign offering free medical examination by an endocrinologist (12). Second, the definition of PCOS used by Diamanti-Kandarakis et al. (12) included only women with oligomenorrhea and hyperandrogenemia. If women with clinical hyperandrogenism, normal androgen levels, and oligomenorrhea were also considered to have PCOS, as in our present study and that by Knochenhauer et al. (11), the prevalence would have been $10.4 \%$ (12). Moreover, as free T was the only androgen measured, the prevalence of PCOS in Lesbos would have been even higher if other serum androgens were measured.

In addition to women with PCOS, Diamanti-Kandarakis et al. (12) found a $29 \%$ prevalence of idiopathic hirsutism in women from Lesbos, an exaggerated figure in view of the prevalences in Madrid, Alabama (11), and elsewhere (16-20). Taken together with the statement of the authors that their recruitment of patients was not fully randomized (12), these high prevalences of PCOS and hirsutism in Lesbos suggest a selection bias in the studied population.

In our present study, women with PCOS and women with hyperandrogenemia and regular cycles were significantly younger than women with idiopathic hirsutism and/or acne without oligomenorrhea or hyperandrogenemia and than women without evidence of hyperandrogenic disorders. The older woman diagnosed with PCOS was 34 yr old in our series and $29 \mathrm{yr}$ old in that by Knochenhauer et al. (11). Further, a recent study reported that the finding of polycystic ovaries by sonography is also much more common in unselected women $35 \mathrm{yr}$ old or younger $(21.6 \%$ prevalence) than in women older than $36 \mathrm{yr}$ (7.8\% prevalence) (6). In agreement, our previous results in a different series of hirsute patients showed that for a similar severity of hyperandrogenic symptoms, younger patients had higher androgen levels of ovarian and/or adrenal origin than older women (22).

These findings may be derived from the well known decline of adrenal androgens with age (23), but might also indicate that age, possibly reflecting the influence of growth factors on adrenal and ovarian function, modulates the clinical and biochemical expression of hyperandrogenism. Definite proof of this point will require studies specifically designed to address this question. Finally, we did not found any influence of obesity or abdominal distribution of fat on the hyperandrogenic disorders studied here.

In conclusion, we found a $6.5 \%$ prevalence of PCOS, as defined by the criteria derived from the NIH/NICHHDsponsored conference in 1990, in a minimally biased popu- lation of Caucasian women from Spain. PCOS and hirsutism are common endocrine disorders in women, especially at younger ages.

\section{Acknowledgments}

We thank Dr. Elena Lasa, Dr. Concepción Zamora, and all the nurses from the Blood Bank of the Hospital Ramón y Cajal (Madrid, Spain) for their collaboration in recruitment and sampling of the population studied. Ms. M. Paz Muñoz kindly helped with hormonal measurements. We also thank DPC-DIPESA (Madrid, Spain) for supplying free of charge the reagents used for T, SHBG, $\mathrm{LH}, \mathrm{FSH}, \mathrm{E}_{2}$, and $\mathrm{P}_{4}$ measurements.

\section{References}

1. Carmina E, Lobo RA. 1999 Polycystic ovary syndrome (PCOS): arguably the most common endocrinopathy is associated with significant morbidity in women. J Clin Endocrinol Metab. 84:1897-1899.

2. Solomon CG. 1999 The epidemiology of polycystic ovary syndrome. Prevalence and associated disease risks. Endocrinol Metab Clin North Am. 28:247-263.

3. Clayton RN, Ogden V, Hodgkinson J, et al. 1992 How common are polycystic ovaries in normal women and what is their significance for the fertility of the population? Clin Endocrinol (Oxf). 37:127-134.

4. Farquhar CM, Birdsall M, Manning P, Mitchell JM, France JT, 1994 The prevalence of polycystic ovaries on ultrasound scanning in a population of randomly selected women. Aust NZ J Obstet Gynecol. 34:67-72.

5. Polson DW, Adams J, Wadsworth J, Franks S. 1988 Polycystic ovaries-a common finding in normal women. Lancet. 1:870-872.

6. Koivunen R, Laatikainen T, Tomas C, Huhtaniemi I, Tapanainen J, Martikainen H. 1999 The prevalence of polycystic ovaries in healthy women. Acta Obstet Gynecol Scand. 78:137-141.

7. Swanson M, Sauerbrei EE, Cooperberg PL. 1981 Medical implications of ultrasonically detected polycystic ovaries. J Clin Ultrasound. 9:219-222

8. Escobar-Morreale H, Pazos F, Potau N, Garcia-Robles R, Sancho JM, Varela C. 1994 Ovarian suppression with triptorelin and adrenal stimulation with adrenocorticotropin in functional hyperadrogenism: role of adrenal and ovarian cytochrome P450c17 $\alpha$. Fertil Steril. 62:521-530.

9. Escobar-Morreale HF, Serrano-Gotarredona J, García-Robles R, Sancho J, Varela C. 1997 Lack of an ovarian function influence on the increased adrenal androgen secretion present in women with functional ovarian hyperandrogenism. Fertil Steril. 67:654-662.

10. Zawadzki JK, Dunaif A. 1992 Diagnostic criteria for polycystic ovary syndrome: towards a rational approach. In: Dunaif A, Givens JR, Haseltine F, Merriam GR, eds. Polycystic ovary syndrome. Boston: Blackwell; 377-384.

11. Knochenhauer ES, Key TJ, Kashar-Miller M, Waggoner W, Boots LR, Azziz R. 1998 Prevalence of the polycystic ovary syndrome in unselected black and white women of the Southeastern United States: a prospective study. J Clin Endocrinol Metab. 83.3078-3082.

12. Diamanti-Kandarakis E, Kouli CR, Bergiele AT, et al. 1999 A survey of the polycystic ovary syndrome in the greek island of Lesbos: hormonal and metabolic profile. J Clin Endocrinol Metab. 84:4006-4011.

13. Hatch R, Rosenfield RL, Kim MH, Tredway D. 1981 Hirsutism: implications, etiology, and management. Am J Obstet Gynecol. 140:815-830.

14. Belchetz PE. 1994 Hormonal treatment of postmenopausal women. N Engl J Med. 330:1062-1071.

15. Azziz R, Hincapie LA, Knochenhauer ES, Dewailly D, Fox L, Boots LR. 1999 Screening for 21-hydroxylase-deficient nonclassic adrenal hyperplasia among hyperandrogenic women: a prospective study. Fertil Steril. 72:915-925.

16. Abraham GE. 1974 Ovarian and adrenal contribution to peripheral androgens during the menstrual cycle. J Clin Endocrinol Metab. 39:240-246.

17. Goldzieher JW, Green JA. 1962 The polycystic ovary. I. Clinical and histologic features. J Clin Endocrinol Metab. 22:325-338.

18. Hartz AJ, Barboriak PN, Wong A, Katayama KP, Rimm AA. 1979 The association of obesity with infertility and related menstrual abnormalities in women. Int J Obes. 3:57-73.

19. McKnight E. 1964 The prevalence of "hirsutism" in young women. Lancet. 1:410-413.

20. Raj SG, Thompson IE, Berger MJ, Taymor ML. 1977 Clinical aspects of the polycystic ovary syndrome. Obstet Gynecol. 49:552-556.

21. Ferriman D, Gallwey JD. 1961 Clinical assessment of body hair growth in women. J Clin Endocrinol Metab. 21:1440-1447.

22. Escobar-Morreale HF, Serrano-Gotarredona J, García-Robles R, Varela C, Sancho JM. 1998 Abnormalities in the serum insulin-like growth factor-I axis in women with hyperandrogenism. Fertil Steril. 70:1090-1100.

23. Parker CRJ, Slayden SM, Azziz R, et al. 2000 Effects of aging on adrenal function in the human: responsiveness and sensitivity of adrenal androgens and cortisol to adrenocorticotropin in premenopausal and postmenopausal women. J Clin Endocrinol Metab. 85:48-54. 